\title{
NOTA
}

\section{MELANOPHORA RORALIS (LINNAEUS, 1758) (DIPTERA: RHINOPHORIDAE) PRIMER REGISTRO DE LA FAMILIA EN URUGUAY}

\author{
María Martínez ${ }^{1 *}$, Silvio Shigueo Nihei $^{2}$ \\ 1 Universidad de la República, Facultad de Ciencias, Sección Entomología, Iguá 4225, CP 11400, Montevideo, \\ Uruguay. \\ 2 Universidade de São Paulo, Instituto de Biociências, Departamento de Zoologia, Rua do Matão, Trav. 14, n. \\ 101, 05508-900, São Paulo, SP, Brazil
}

*Autor para correspondencia: María Martínez. mm@fcien.edu.uy

\section{RESUMEN}

Rhinophoridae (Diptera: Oestroidea) es una pequeña familia de dípteros caliptrados con alrededor de 150 especies y 24 géneros en el mundo. Siete géneros y 24 especies se distribuyen en el continente americano. Se registra por primera vez a la especie paleártica Melanophora roralis (Linnaeus, 1758) en Uruguay, basada en especímenes adultos capturados en Montevideo, en 2018. Sus larvas son endoparásitas en isópodos terrestres (Crustacea).

Palabras Clave: Especie introducida, endoparásita, isópodos terrestres.

\section{ABSTRACT}

Melanophora roralis (Linnaeus, 1758) (Diptera: Rhinophoridae) family first registry in Uruguay. Rhinophoridae (Diptera: Oestroidea) is a small family of calyptrate dipterans with around 150 species and 24 genera in the world. Seven genera and 24 species are distributed in the American continent. The palearctic species Melanophora roralis (Linnaeus, 1758) in Uruguay is recorded for the first time, based on adult specimens captured in Montevideo in 2018. Its larvae are endoparasitic in terrestrial isopods (Crustacea).
Key words: Introduced species, endoparasite, terrestrial isopods.

Los rinofóridos comprenden una pequeña familia de dípteros caliptrados, pertenecientes a la superfamilia Oestroidea, con alrededor de 24 géneros y 150 especies descritas para el mundo (Crosskey, 1977; Wood, 1987). Hasta el momento, se han registrado siete géneros y 24 especies en el continente americano (Tabla 1), 21 de las cuales son nativas: Apomorphyto inbio Cerretti, Lo Giudice \& Pape, 2014, 16 especies de Bezzimyia Townsend, 1919, Shannoniella cuspidata Townsend, 1939, Shannoniella setinervis Nihei, Andrade, Pape \& Cerretti, 2016, Trypetidomima lutea Townsend, 1935, y Trypetidomima fusca Nihei \& Andrade, 2014. Las especies Melanophora roralis (Linnaeus, 1758), Phyto discrepans (Pandellé, 1896) y Stevenia deceptoria (Loew, 1847) han sido introducidas en el continente americano (Mulieri et al., 2010; Nihei \& Andrade, 2014; Nihei et al., 2016).

La biología de las especies de rinofóridos de las cuales existe información relevante, son parasitoides obligados de isópodos terrestres en la etapa larval (Nihei \& Andrade, 2014). En la presente nota se cita por primera vez la presencia de Melanophora roralis (Linnaeus, 1758) (Diptera: Rhinophoridae) en Uruguay.

Tabla 1. Géneros de Rhinophoridae y número de especies registradas en el continente americano.

\begin{tabular}{lccc} 
Género & Autor & No spp. & Distribución en el continente americano \\
\hline Apomorphyto & Cerretti, Lo Giudice \& Pape, 2014 & 1 & Costa Rica \\
Bezzimyia & Townsend, 1919 & 16 & EEUU hasta Brasil \\
Melanophora & Meigen, 1803 & 1 & EEUU hasta Argentina \\
Phyto & Robineau-Desvoidy, 1830 & 1 & Canadá \\
Shannoniella & Townsend, 1939 & 2 & Brasil \\
Stevenia & Robineau-Desvoidy, 1830 & 1 & Argentina \\
Trypetidomima & Townsend, 1935 & 2 & Brasil
\end{tabular}


Adultos de Rhinophoridae (cuatro $\hat{o}$, tres $q$ ) fueron colectados manualmente con un tubo de ensayo de $50 \mathrm{ml}$, en Montevideo, Uruguay, 34052'43"S, 5608'09'W, posados en las paredes externas de vivienda, en el jardín, en el barrio Unión, el $20 / 1 / 2018$. Para la identificación se utilizaron las claves de Cerretti \& Pape (2009) y Mulieri et al. (2010). El material examinado durante este estudio se depositó en las colecciones de la sección Entomología, Facultad de Ciencias, Universidad de la República, Uruguay (FCE-Dip 0366) y en el Museu de Zoologia da Universidade de São Paulo, Brazil (MZSP).

Melanophora roralis es una especie paleártica introducida en el continente americano, con registros en Estados Unidos de América (Brues, 1903; Tucker, 1906; Jones, 1948), Brasil (Parker, 1953; Guimarães, 1971, 1977), Argentina (Parker, 1953; Blanchard \& De Santis, 1975), Jamaica (Crosskey, 1977) y Chile (González, 1998; Cepeda et al., 2006). Los adultos son de tamaño medio con una longitud corporal de 4.0 a $7.1 \mathrm{~mm}$ y de color general negruzco o pardusco. La hembra presenta alas castañas con el ápice más claro o hialino (Fig. 1). La vena $\mathrm{M}$ del ala es completa y curvada en sentido anterior, uniéndose a la vena $\mathrm{R} 4+5$ antes del margen del ala, siendo la celda $r 4+5$ cerrada y el pecíolo largo (Figs. 1, 2). La cerda escutelar apical cruzada está presente y bien desarrollada (Crosskey, 1977; Cerretti \& Pape, 2009).

Las larvas de $M$. roralis son endoparasitarias de isópodos terrestres, conocidos comúnmente como "cochinillas", "bicho bolita" o "bicho de la humedad" (Crustacea: Isopoda: Oniscidea). En Uruguay se han registrado varias especies de isópodos, entre ellas: Armadillidium vulgare (Latreille, 1804), Armadillidium nasatum Budde-Lund, 1885, Porcellio

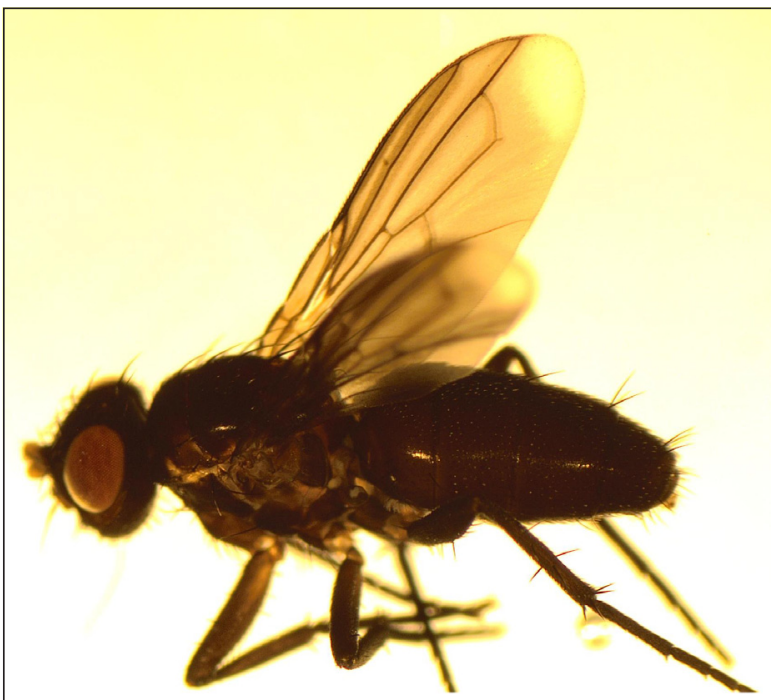

Fig. 1. Melanophora roralis, hembra vista lateral. laevis Latreille, 1804 y Balloniscus sellowi (Brandt, 1833) (Waller \& Verdi, 2017) pero en ninguna de ellas se ha detectado parasitosis producida por larvas de $M$. roralis (Waller com. pers.). Esta especie de rinofórido deposita sus huevos en el sustrato húmedo frecuentado por isópodos, como hojarasca y madera en descomposición. Después que el primer estadio larvario de la mosca eclosiona, busca un huésped al cual parasitar. Una sola larva se desarrolla dentro de cada huésped, alimentándose y mudando, hasta quedar solo el exoesqueleto vacío del hospedero con el pupario del rinofórido dentro, generalmente, con el extremo anterior dirigido hacia el extremo anterior del huésped. Pasa el invierno en estado inmaduro, los adultos emergen en verano y pueden observarse en jardines de áreas urbanas (Wood, 1987).Según Sassaman \& Garthwaite (1984) la longevidad de los adultos y la fecundidad de las hembras de $M$. roralis fueron funciones directas del tamaño de los huéspedes, Porcellionides pruinosus (Brandt, 1833) y Porcellio scaber Latreille, 1804, y puede reflejar adaptaciones en este díptero para su persistencia a bajas densidades de población.

Si bien la introducción de $M$. roralis en el continente sudamericano fue detectada en la década del 50 (Parker, 1953), hasta ahora no se había citado para Uruguay. Con el registro de esta especie, se adiciona una nueva familia a la fauna de Diptera de Uruguay. Se presume que existe un número mayor de especies de esta familia en el país. Un ejemplo que hace suponer esta presunción es otra especie introducida, S. deceptoria, registrada en Buenos Aires, Argentina (Mulieri et al., 2010). Según Nihei (2016) hay nuevos taxones de Rhinophoridae para ser descritos en la fauna Neotropical. Recientemente fueron identificadas nuevas especies en estados densamente poblados como San Pablo y Río de Janeiro, Brasil (Nihei \& Andrade, 2014; Nihei et al., 2016), evidenciando que los estudios de esta Familia, en América del Sur, son incipientes.

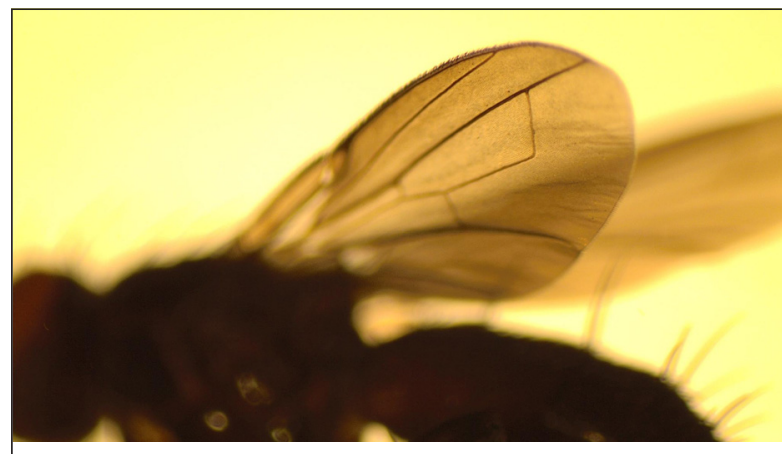

Fig. 2. Melanophora roralis, macho vista lateral, detalle del ala. 


\section{AGRADECIMIENTOS}

Deseamos expresar nuestro agradecimiento a $\mathrm{F}$. Pérez-Miles, C. Perafán y A. Waller (Entomología, Facultad de Ciencias, Uruguay) por la colaboración brindada. S.S. Nihei agradece por el financiamiento de su investigación a FAPESP (proc. n. 2015/107880) y CNPq (403165/20164) y la beca de productividad científica de CNPq (proc. n. 303615/2015-2). También agradecemos a los revisores y al editor por las sugerencias efectuadas que enriquecieron el manuscrito.

\section{REFERENCIAS}

Blanchard E.E. \& De Santis L. 1975. Primera lista anotada de Oestromuscarios entomófagos argentinos. Revista de Investigaciones Agropecuarias, Serie V, Patología Vegetal, 12: 7-76.

Brues C.T. 1903. A dexiid parasite of the sow-bug. Entomological News, 14: 291.

Cepeda J., Pola M., Zuleta C. \& González C. 2006. Relaciones de abundancia y diversidad de la entomofauna del Humedal Tambo-Puquíos. En: J. Cepeda (Ed.). Geoecología de los Andes desérticos: la alta montaña del Valle del Elqui, pp. 475-521. Ed. Universidad de La Serena, La Serena, Chile.

Cerretti P.H. \& Pape T. 2009. Phylogeny and redefinition of the genus Melanophora (Diptera: Rhinophoridae), with description of a new species from Sardinia. Zootaxa, 2318: 552-565.

Crosskey R.W. 1977. A review of the Rhinophoridae (Diptera) and a revision of the Afrotropical species. Bulletin of the British Museum of Natural History (Entomology), 36: 1-66.

González C. 1998. Primer registro para Chile de Melanophora roralis (Linnaeus) (Diptera: Rhinophoridae). Acta Entomológica Chilena, 22: 111-112.

Guimarães J.H. 1971. Family Tachinidae (Larvaevoridae). En: N. Papavero (Ed.) A Catalogue of the Diptera of the Americas South of the United States. Departamento de Zoologia, Secretaria da Agricultura, São Paulo, Brasil, 104: 1-333
Guimarães J.H. 1977. Host-parasite and parasite-host catalogue of South American Tachinidae (Diptera). Arquivos de Zoologia, 28: 1-131.

Jones F.M. 1948. Notes on Melanophora roralis (Linn.) (Diptera). Psyche: A Journal of Entomology, 55: 31-34.

Mulieri P.R., Patitucci L.D., Mariluis J.C. \& Pape T. 2010. Long-distance introduction: first New World record of Stevenia deceptoria (Loew) and a key to the genera of New World Rhinophoridae (Diptera). Zootaxa, 2524: 66-68.

Nihei S.S. 2016. Family Rhinophoridae. En: Wolff M., Nihei S.S. \& de Carvalho C.J.B. (Eds.). Catalogue of Diptera of Colombia. Zootaxa, 4122: 881-883.

Nihei S.S. \& Andrade M.R. 2014. Revision of Trypetidomima (Diptera: Rhinophoridae) with description of a new brazilian species. Florida Entomologist 97:724-733.

Nihei S.S., Andrade M.R, Pape T. \& Cerretti P. 2016. The Shannoniella sisters (Diptera: Rhinophoridae). Zootaxa, 4061: 85-92.

Parker H.L. 1953. Miscellaneous notes on South American dipterous parasites. Bolletino Laboratorio di Entomologia Agraria "Filippo Silvestri" di Portici, 12: 45-73.

Sassaman C. \& Garthwaite R. 1984. The Interaction between the Terrestrial Isopod Porcellio scaber Latreille and One of its Dipteran Parasites, Melanophora roralis (L.) (Rhinophoridae). Journal of Crustacean Biology, 4: 595-603.

Tucker E.S. 1906. Contributions towards a catalogue of insects of Kansas. Results of personal collecting. Transactions of the Kansas Academy of Science, 20: 190-201.

Waller A. \& Verdi A. 2017. Community of terrestrial isopods (Crustacea,Oniscidae): Differences between natural and labored environments. Journal of Entomology and Zoology Studies, 5: 1102-1107.

Wood D.M. 1987. Rhinophoridae. En: J.F. McAlpine (Ed.) Manual of Nearctic Diptera. Agriculture Canada, Ottawa, 2: 1187-1191.

Fecha de Recibido: 10 de mayo de 2018 Fecha de Aceptado: 12 de noviembre de 2018 